\title{
Traumatic epidural pneumorrhachis: a case report
}

\author{
YY Yang ${ }^{1}$, MB, BS, CB Chua ${ }^{1}$, MD, CW Hsu ${ }^{1,2}, \mathrm{MD}, \mathrm{PhD}, \mathrm{KH} \mathrm{Lee}^{1,2 *}$, MD, PhD \\ ${ }^{1}$ Department of Emergency Medicine, E-Da Hospital, I-Shou University, Kaohsiung, Taiwan \\ ${ }^{2}$ School of Medicine for International Students, I-Shou University, Kaohsiung, Taiwan
}

*Corresponding author: peter1055@gmail.com

Hong Kong Med J 2020;26:528-31

https://doi.org/10.12809/hkmj208431

\section{Case report}

A 62-year-old man with no known systemic disease was brought to an emergency department by ambulance. He was found trapped in a car after colliding with a bridge and was found to have been driving under the influence of alcohol. On arrival, he complained of headache, neck pain, and chest pain. Physical examination revealed blood pressure of $133 / 71 \mathrm{~mm} \mathrm{Hg}$, heart rate 71 beats per minute, respiratory rate 22 breaths per minute, and oxygen saturation on air of $93 \%$. He appeared intoxicated and was disorientated with a score of 13 (E3V4M6) on the Glasgow Coma Scale. His pupil size was $3.5 \mathrm{~mm}$ with bilateral normal reaction to light. A thorough neurological examination could not be performed but the patient was able to move all four limbs without limitation. Nonetheless a scalp laceration, upper back tenderness and left lower cervical crepitus were evident during palpation. Chest examination revealed bilateral equal breath sounds but some basal crackles and a left flank abrasion about $3 \times 4 \mathrm{~cm}$ in size. Haemogram and biochemistry test results were within normal limits but serum alcohol level was elevated at $2.48 \mathrm{~g} / \mathrm{L}$. Chest radiography revealed a posterior fracture at the left fourth to sixth ribs and right ninth rib as well as left cervical subcutaneous emphysema that raised the suspicion of barotrauma associated with chest trauma (Fig 1). In view of the possibility of barotrauma and intracranial injuries, further evaluation with computed tomography (CT) of the brain and chest was arranged. The former was normal and the latter confirmed right first to second posterior ribs and left third to sixth posterior ribs fractures, as well as a small left-sided pneumothorax, bilateral pulmonary contusions, and pneumomediastinum. Notably, free air accumulated in the posterior epidural spaces of the cervical and thoracic spine as well as in the left retrospinal and paraspinal muscle layers with accompanying left second and third thoracic spine posterior neural arch fracture in cervical spine CT scan (Fig 2); these findings were compatible with epidural pneumorrhachis. Additionally, air was seen dissecting from the posterior neck into the epidural space. A neurosurgeon recommended close observation rather than surgical intervention for both the epidural pneumorrhachis and thoracic spine fracture. The patient was admitted to the thoracic surgical ward where he received conservative therapy for rib fractures, pneumothorax and pneumomediastinum including meperidine for pain control and oxygen therapy via a nasal cannula at $3 \mathrm{~L} / \mathrm{min}$. New chest radiographs were taken on day 4 after surgery. Cervical emphysema had mostly resolved but there was progression of pulmonary contusion with bilateral minimal pleural effusions. The patient also exhibited wheezing and dyspnoea that warranted inhaled bronchodilators and systemic steroid therapy. The patient was discharged on day 12 after surgery with symptom improvement; no neurological sequelae were noted during follow-up at the out-patient department.

\section{Discussion}

Pneumorrachis (PRS) is characterised by the presence of air within the spinal canal and can be classified according to its location as subarachnoid or epidural PRS. Each is associated with different pathophysiological mechanisms and causes. It may result non-traumatically from spinal degeneration, gas-producing infections, or spontaneous pneumomediastinum in patients with asthma. Iatrogenic PRS may occur following a surgical procedure, epidural anaesthesia, or lumbar puncture but PRS following trauma is rare. ${ }^{1}$ Almost all types of PRS are found in association with air distribution in other compartments and cavities of the body. For example, PRS is observed in conjunction with pneumocephalus, pneumothorax, pneumomediastinum, pneumopericardium, or subcutaneous emphysema. Pneumorrachis following trauma is rare and arises from the presence of air in the posterior mediastinum or retropharyngeal space dissecting along the fascial planes from the posterior mediastinum or retropharyngeal space through the neural foramina into the epidural space under the driving pressure of a pneumothorax and pneumomediastinum and low resistance of loose connective tissue. ${ }^{2}$

Epidural PRS has been reported to 


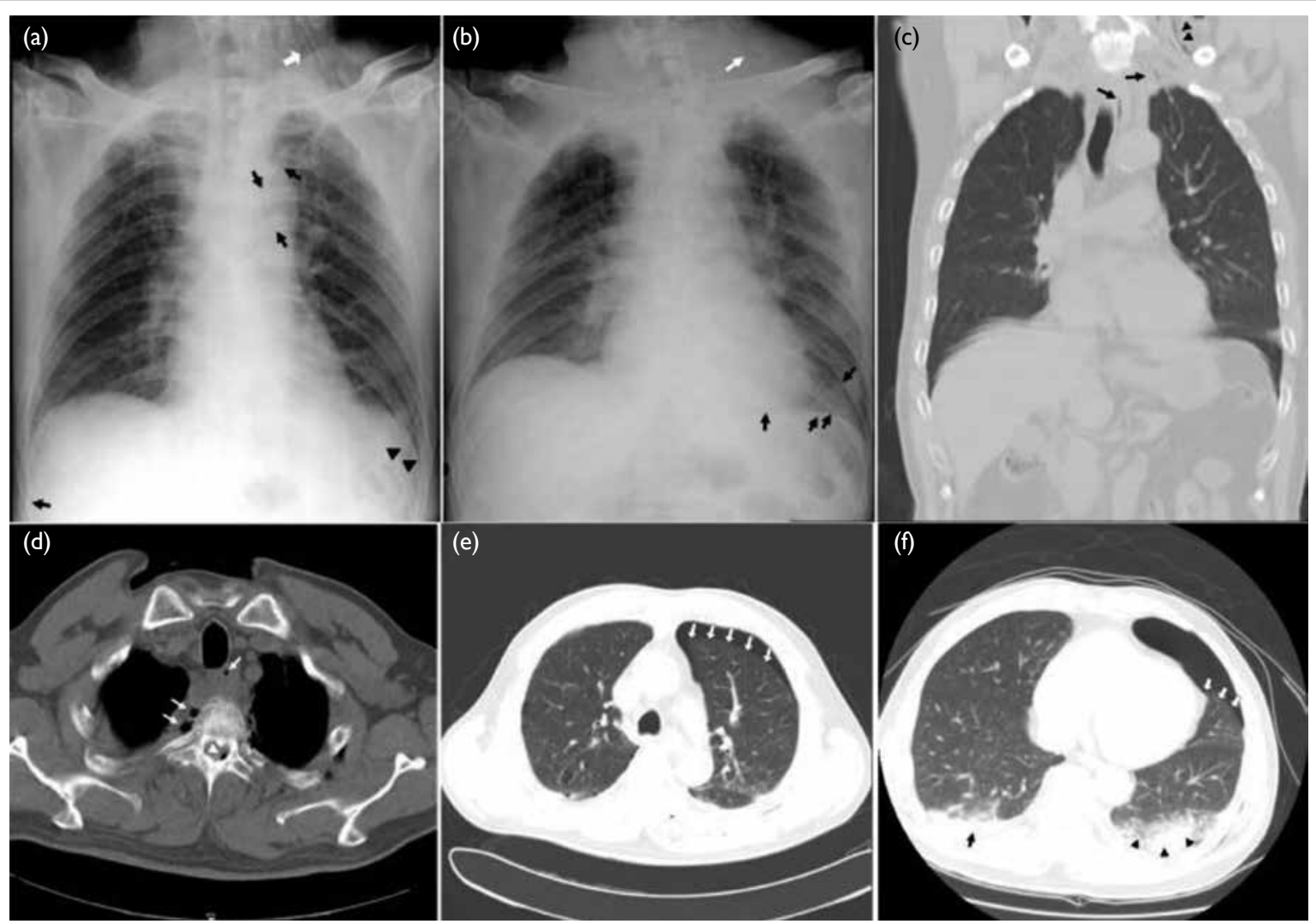

FIG I. (a) Chest radiograph anteroposterior view showing left cervical subcutaneous emphysema (white arrow) and posterior fracture of left fourth to sixth ribs and right ninth rib (black arrows) and deep sulcus sign (black arrowheads). (b) New chest radiograph anteroposterior view on day 4 showing near total resolution of left cervical subcutaneous emphysema (white arrow) but left lower pulmonary contusion with progression (black arrows). (c) Non-contrast thoracic computed tomography (CT) coronal view in lung window setting displaying left cervical subcutaneous emphysema (black arrowheads) and pneumomediastinum (black arrows). (d) Non-contrast thoracic CT axial view in bone window setting demonstrating pneumomediastinum (white arrows) and epidural pneumorrhachis (white arrowhead). (e) and (f) Non-contrast thoracic CT axial view in lung window setting demonstrating left-sided small pneumothorax (white arrows) and left pulmonary contusion (black arrowheads) and right minimal pleural effusion (black arrow)

occur most commonly secondary to traumatic pneumomediastinum. Neurological deficits have not been reported in epidural PRS secondary to trauma. In contrast, traumatic subarachnoid PRS is almost always secondary to major trauma and skull bone fractures, and indicates severe injury and possible association with complications such as tension pneumocephalus or meningitis. ${ }^{3}$ Various neurological deficits, such as acute lumbar root compression, chronic radiculopathy, and cauda equina syndrome may occur. It is therefore important to identify air in the epidural space and subarachnoid space to enable prompt and adequate management. ${ }^{4}$ The possible mechanism of PRS in our patient was air entry into the epidural space because of pneumomediastinum, left-sided pneumothorax and subcutaneous emphysema extending through the fractured neural arch of the thoracic spines into the epidural space; these conditions fulfil the criteria for benign traumatic PRS. Criteria for a diagnosis of benign traumatic epidural PRS include substantial pneumomediastinum with extensive subcutaneous emphysema due to thoracic trauma. ${ }^{4}$ There may also be incidental findings of small amounts of epidural air demonstrable only on CT and unrelated to spinal trauma. "Benign" epidural PRS can be managed conservatively with spontaneous resolution of epidural air. ${ }^{4}$ Repeat or further neuroimaging studies are not routinely provided in cases of epidural PRS, provided there is no new or progressive neurological deficit. Three cases have been reported with complete resolution of PRS on follow-up CT scan after 4 to 14 days. ${ }^{5-7}$

The PRS is usually asymptomatic and an accidental finding on $\mathrm{CT}$ images while evaluating other severe or life-threatening torso injuries. Although CT is the gold standard for diagnosis, it is difficult to differentiate epidural and subarachnoid PRS. Nonetheless in the former, air is collected at the corner or peripherally unlike subarachnoid PRS 


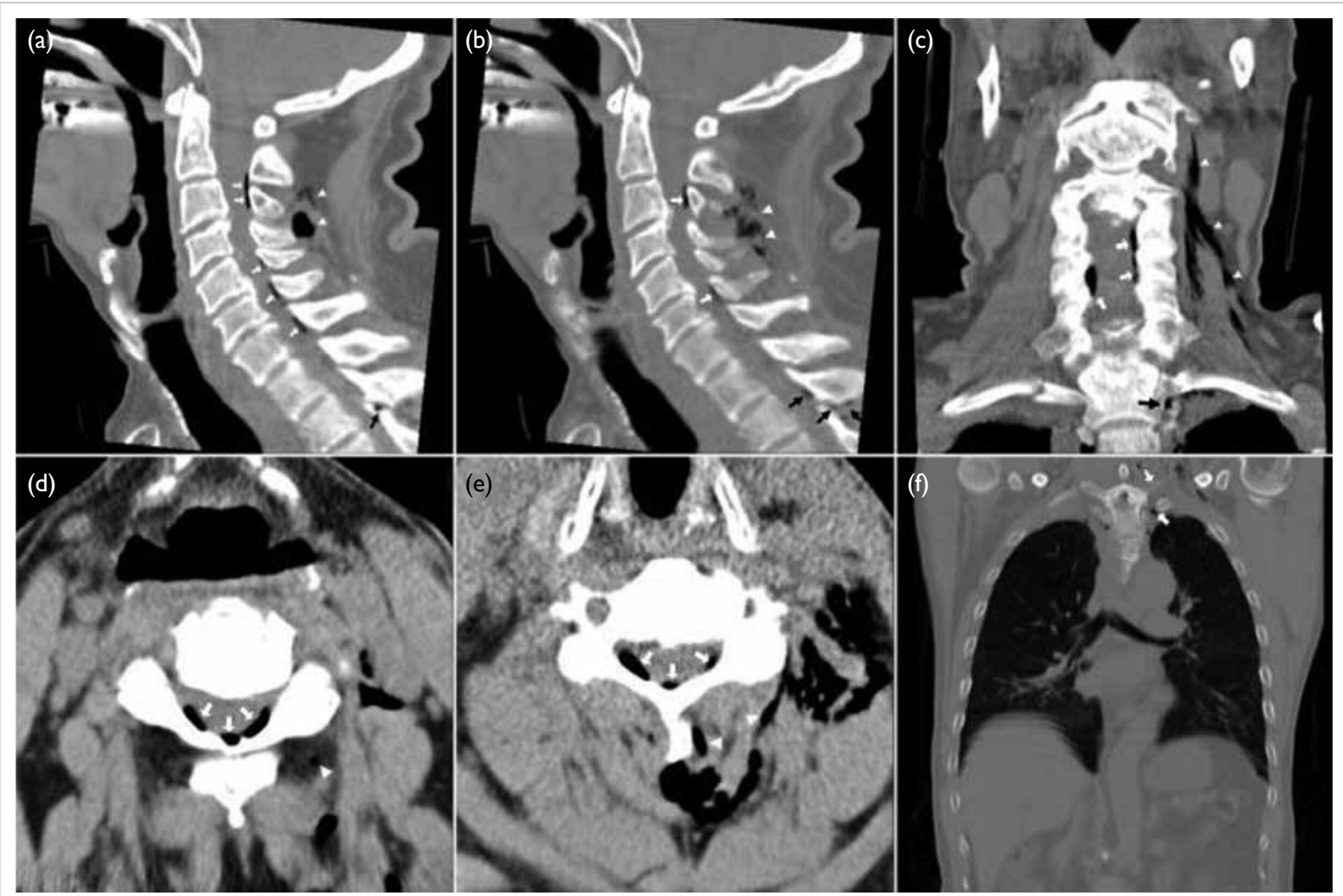

FIG 2. (a) Cervical spine computed tomography (CT) scan sagittal view in soft tissue window setting revealing air distributed in the epidural space (epidural pneumorrachis) at C-spine C3, C5 to C7 level (white arrows), posterior cervical subcutaneous emphysema (white arrowheads) and (black arrow) pointing out air dissecting from posterior neck into the epidural space; free air within the epidural space is located at the corner or peripherally. (b) Cervical spine CT scan sagittal view in bone window setting demonstrating epidural pneumorrhachis at C-spine C3 and C6 level (white arrows), posterior cervical subcutaneous emphysema (white arrowheads) and (black arrows) pointing out air dissecting from posterior neck into epidural space at the level of T2. (c) Cervical CT coronal view revealing epidural pneumorrhachis (white arrows), left cervical subcutaneous emphysema (white arrowheads) and pneumomediastinum (black arrow). (d) and (e) Cervical spine CT scan axial view at C3 level and C5 level in soft tissue window setting demonstrating epidural pneumorrhachis (white arrows) and air within the prevertebral layer of deep cervical fascia, especially in the paraspinal portion (white arrowheads). (f) Non-contrast thoracic CT coronal view in bone window setting showing air entry through the fractured neural arch of the left thoracic spine T2-3 resulting in epidural pneumorrhachis (white arrows)

where it is distributed more centrally in the canal based on normal anatomy. Magnetic resonance imaging or intrathecal contrast CT can distinguish intradural from extradural air. ${ }^{7}$ In most cases of epidural PRS, the air reabsorbs spontaneously without recurrence and the patient can be managed conservatively. ${ }^{1}$ In subarachnoid PRS, timely consultation with a neurosurgeon for decompression of nerve root injuries, tension pneumocephalus and exploratory surgery by a chest surgeon to detect severe thoracic trauma is vital. Impressively, Avci et $\mathrm{al}^{5}$ reported a case of complete resolution of subarachnoid PRS on the fifth day of admission with recovery of neurological deficit after chest tube insertion. They concluded that the negative pressure of the chest drain to withdraw the air supported the hypothesis of a one-way valve mechanism in which high pressure air travels through fascial layers but is unable to return. The routine use of antibiotic is not recommended in either type unless meningitis develops. ${ }^{1}$

\section{Conclusion}

Traumatic PRS is rare and an incidental radiological finding. Epidural PRS is often self-limiting with a good prognosis. Although most patients can be managed conservatively, prompt recognition of subarachnoid PRS with neurological deficits and other concurrent life-threatening torso injuries is vital.

\section{Author contributions}

Concept or design: KH Lee.

Acquisition of data: YY Yang.

Analysis or interpretation of data: $\mathrm{CB}$ Chua.

Drafting of the manuscript: YY Yang and KH Lee.

Critical revision of the manuscript for important intellectual content: CW Hsu. 
All authors had full access to the data, contributed to the study, approved the final version for publication, and take responsibility for its accuracy and integrity.

\section{Conflicts of interest}

All authors have disclosed no conflicts of interest.

\section{Acknowledgement}

We would like to extend our special thanks to Dr Cheng-yang Lee, Department of Medical Imaging, Chi Mei Medical Center, Tainan, Taiwan who provided insight and expertise on the image interpretation that greatly improved our manuscript.

\section{Funding/support}

All authors have no commercial association, such as consultancies, stock ownership or other equity interests or patent-licensing arrangements.

\section{Ethics approval}

The patient was treated in accordance with the tenets of the Declaration of Helsinki. The patient provided written informed consent for all treatments and procedures.

\section{References}

1. Oertel MF, Korinth MC, Reinges MH, Krings T, Terbeck S, Gilsbach JM. Pathogenesis, diagnosis and management of pneumorrhachis. Eur Spine J 2006;15 Suppl 5:636-43.

2. Defouilloy C, Galy C, Lobjoie E, Strunski V, Ossart M. Epidurual pneumatosis: A benign complication of benign pneumomediastinum. Eur Respir J 1995;8:1806-7.

3. Goh BK, Yeo AW. Traumatic pneumorrhachis. J Trauma 2005;58:875-9.

4. Willing SJ. Epidural pneumatosis: a benign entity in trauma patients. AJNR Am J Neuroradiol 1991;12:345.

5. Avci İ, Başkurt O, Şirinoğlu D, Aydin MV. Rapid disappearance of pneumorrhachis after chest tube placement. Turk J Emerg Med 2019;19:146-8.

6. Kim SW, Seo HJ. Symptomatic epidural pneumorrhachis: a rare entity. J Korean Neurosurg Soc 2013;54:65-7.

7. Pfeifle C, Henkelmann R, von der Höh N, et al. Traumatic pneumorrhachis. Injury 2020;51:267-70.

\section{Answers to CME Programme Hong Kong Medical Journal October 2020 issue}

Hong Kong Med J 2020;26:413-20

I. Labour analgesia: update and literature review
A
1. True
2. True
3. False
4. False
5. True
B
1. True
2. True
3. False
4. True
5. True

Hong Kong Med J 2020;26:421-31

II. Update to the Hong Kong Epilepsy Guideline: evidence-based recommendations for clinical management of women with epilepsy throughout the reproductive cycle
A
1. True
2. False
3. False
4. False
5. False
B
1. True
2. False
3. True
4. True
5. True 